\title{
ASPECTOS CLÍNICOS E EXPERIMENTAIS DA DOR EM EQUINOS: REVISÃO DE LITERATURA
}

Recebido: 25/10/2015

\author{
ALVES, José Edgard de Oliveira ${ }^{1}$; \\ VIEIRA, Evelyn Mayara Perrut ${ }^{2}$; \\ SARTORI, Fábio ${ }^{3}$; \\ CATELLI, Marcelo Flores ${ }^{3}$.
}

Aceito: 06/06/2016

${ }^{2}$ Acadêmica de Medidente de Anestesiologia Veterinária da Universidade Estadual do Norte Fluminense, Sombra.

\section{RESUMO}

\begin{abstract}
A dor é um fenômeno produzido a partir de uma experiência sensorial ou emocional aversiva, representada pela consciência de um dano tecidual real ou potencial, que projeta uma série de alterações fisiológicas e comportamentais que visam evitar ou minimizar a ocorrência de tal dano com o intuito de proteger o organismo e manter a homeostase. Este fenômeno tem impacto direto sobre o bem-estar animal e seu controle é uma responsabilidade profissional do Médico Veterinário. A percepção da dor pelo indivíduo depende de uma série de eventos que envolvem o sistema nervoso periférico e central, tais como a transdução, transmissão, modulação, projeção e processamento central do impulso elétrico gerado pelo estímulo nocivo. Atualmente, diversos fármacos estão disponíveis para o emprego no controle da dor na medicina equina e vários métodos experimentais são descritos para o estudo da dor nesta espécie. Estes modelos permitem avaliar a eficácia de fármacos analgésicos e aprofundar a compreensão sobre os mecanismos envolvidos na fisiopatologia da dor. O objetivo deste artigo é abordar os principais desafios no estudo da dor em equinos, tanto sob o ponto de vista experimental quanto clínico.
\end{abstract}

Palavras-chave: Dor. Analgesia. Nocicepção. Modelos Experimentais. 


\section{INTRODUÇÃO}

A dor é um aspecto determinante para as condições de saúde e bem-estar animal e seu controle por parte do Médico Veterinário reflete um comportamento ético e enfatiza uma responsabilidade profissional (BORGES, 2010). Nas últimas décadas, com a melhor compreensão dos mecanismos da dor e de seu controle, houve um aumento crescente na preocupação com a analgesia na Medicina Veterinária. Grandes avanços como a descoberta de novas moléculas e fármacos possibilitaram maior eficiência no controle da dor (MELO JÚNIOR et al., 2012).

A dor é definida pela Associação Internacional para o Estudo da Dor como uma experiência sensorial e emocional desagradável associada a um dano tecidual real ou potencial ou descrita nos termos de tal dano (GRAUW; VAN LOON, 2016). Um conceito mais apropriado para definir a dor em animais seria o citado por Muir (2010), o qual refere-se à dor como uma experiência sensorial ou emocional aversiva, representando consciência de dano ou ameaça à integridade tecidual pelo animal, que provoca mudanças fisiológicas e comportamentais objetivando evitar tal dano, reduzir a probabilidade de recorrência deste dano e promover a recuperação.

Nota-se que esta definição é mais complexa em se tratando da dor animal, na qual a parte emocional e psicológica apresenta grande dificuldade de avaliação e os animais não são capazes de expressar verbalmente suas sensações (POLYDORO, 2006).

O reconhecimento da dor por parte do Médico Veterinário contribui para melhor aproveitamento e eficiência na instituição de protocolos analgésicos. Todavia, a experiência e a demonstração dos sinais de dor nos animais sofre influência de fatores como raça, indivíduo, ambiente e medicamentos administrados, entre outros fatores (MELO JÚNIOR et al., 2012). Em outras palavras, os sinais comportamentais da dor nos pacientes em Medicina Veterinária dependem de uma série de características particulares de cada espécie animal.

Diante destas informações, o presente artigo teve como objetivo revisar os aspectos gerais da dor em equinos, destacando-se os principais desafios clínicos e experimentais no reconhecimento, avaliação e no controle deste fenômeno. 


\section{DOR EM EQUINOS}

\section{Fisiopatologia da dor}

Podemos classificar a dor como fisiológica ou patológica. A primeira refere-se à dor relacionada às respostas protetoras, tais como reflexo de retirada ou a reação de fuga, que visam interromper o estímulo doloroso. A dor patológica ocorre quando um estímulo não é transitório (KLAUMANN et al., 2008) e está associada a condições inflamatórias, como lacerações e artrites, por exemplo; ou neuropáticas, como traumas neurológicos, neuroma e infecções virais, por exemplo (MUIR, 2010). Temporalmente, podemos classificar a dor como aguda, quando sua ocorrência é recente, ou crônica, quando a duração é prolongada (KLAUMANN et al., 2008).

O primeiro passo na sequência dos eventos que originam o fenômeno sensitivo-doloroso é a transformação dos estímulos ambientais físicos ou químicos em potenciais de ação, que são transferidos das fibras nervosas periféricas para o sistema nervoso central (TEIXEIRA, 2001). Os estímulos nocivos são os responsáveis por desencadear todo o processo de nocicepção. São eles os responsáveis por lesar os tecidos e causar a dor (CASTRO, 2007; LE BARS et al., 2001). A nocicepção compreende uma série de eventos que envolve a transdução, transmissão, modulação, projeção e processamento central do dano tecidual real ou potencial (MUIR, 2010).

A via aferente da nocicepção é composta por uma cadeia de três neurônios: (1) o neurônio de primeira ordem, originado na periferia e que se projeta para a medula espinhal; (2) o neurônio de segunda ordem, que ascende pela medula espinhal; e (3) o neurônio de terceira ordem, que se projeta para o córtex cerebral (KLAUMANN et al., 2008). Os nociceptores também se dividem em subtipos: existem os (1) mecanotermonociceptores A-delta; (2) mecanonociceptores A-delta de alto limiar; e (3) nociceptores $C$ polimodais de baixo e de alto limiar (CASTRO, 2007). As fibras A-delta transmitem os impulsos nervosos mais rapidamente que as fibras tipo $\mathrm{C}$ e são responsáveis pela resposta aguda que desencadeia aversão e o reflexo de retirada. A ativação das fibras do tipo C dá início ao fenômeno 
conhecido como segunda dor, por resultar em um início mais tardio em relação ao estímulo nas fibras A-delta (MUIR,2010). As fibras aferentes de primeira ordem formam conexões diretas ou indiretas com uma das três populações de neurônios do corno dorsal da medula: (1) interneurônios, subdivididos em excitatórios e inibitórios; (2) neurônios proprioespinhais, que estendem-se por múltiplos segmentos espinhais e estão envolvidos com a atividade reflexa; (3) neurônios de projeção, que participam na transmissão rostral através da medula espinhal até centros supraespinhais como o mesencéfalo e córtex. Os três componentes são interativos e são essenciais para o processamento da informação nociceptiva, o que facilita a geração de uma resposta à dor apropriada e organizada (KLAUMANN et al., 2008).

Quando os nociceptores são persistentemente estimulados, ocorre um aumento da sensibilidade e uma redução do limiar para um estímulo, acarretando em um aumento da intensidade e da duração das respostas ao estímulo nocivo. Esse fenômeno constitui a sensibilização periférica aos estímulos nocivos e dá origem à inflamação (CASTRO, 2007). A sensibilização central se dá quando aumenta a excitabilidade e a responsividade dos neurônios nociceptivos centrais do corno dorsal para estímulos aferentes abaixo de um limiar nociceptivo normal. Este processo envolve a ativação de receptores N-Metil-DAspartato (Muir, 2010). A modulação da dor consiste na ativação de interneurônios inibitórios locais pelos estímulos nociceptivos que chegam ao corno dorsal da medula, os quais formam sinapses recíprocas com os neurônios aferentes primários e, em certos casos, com os neurônios ascendentes secundários, criando uma espécie de feedback inibitório (TEIXEIRA, 2010) que envolve a participação de opióides endógenos (CASTRO, 2007).

Todos os eventos supracitados são refletidos clinicamente através de respostas neuroendócrinas, representadas por sinais como o aumento do tônus simpático, vasoconstrição, aumento da resistência vascular sistêmica, aumento da frequência e do débito cardíaco, redução do tônus gastrointestinal e urinário e aumento do tônus musculoesquelético, hipoventilação, aumento no tempo de coagulação sanguínea, fibrinólise e agregação plaquetária, aumento da secreção de catecolaminas, ACTH, cortisol, $\mathrm{ADH}$, hormônio do crescimento, renina, angiotensina II, aldosterona e glucagon, com redução da liberação de insulina e testosterona (KLAUMANN et al., 2008). 


\section{Reconhecimento e Avaliação da Dor}

A ausência de comunicação verbal é, sem dúvida, um dos maiores obstáculos para a avaliação da dor em animais. Existem situações em que se torna mais fácil realizar o reconhecimento de um animal em condições de dor, como, por exemplo, quando há vocalização na resposta a um estímulo nocivo. Por outro lado, certificar-se de que um animal não está sentindo dor em um dado momento pelo fato de não apresentar sinais físicos típicos ou comportamentais é um processo ainda bastante dificultoso (LE BARS et al., 2001). A percepção da dor por parte do clínico contribui para a instalação de protocolos analgésicos mais eficientes. Entretanto, por se tratar de uma experiência subjetiva, os critérios para sua mensuração são complexos, sendo necessária a criação e a padronização de métodos eficientes de avaliação (MELO JÚNIOR et al., 2012).

A dor pode ser avaliada de modo subjetivo ou objetivo. O primeiro se refere à análise e interpretação do comportamento animal e em sistemas de escores de dor (GRAUW; VAN LOON, 2016; SILVA JÚNIOR, 2009). Exemplos de sistemas de escores de dor incluem a escala analógica visual, a escala de classificação numérica, escala descritiva simples e o método de análise estimativa de atividades, que consiste na monitoração e contabilização por um longo período de comportamentos que normalmente se apresentam alterados em condições de dor (GRAUW; VAN LOON, 2016). Todavia, estes podem variar de acordo com uma série de fatores, fazendo com que a interpretação única da dor seja bastante difícil (TAYLOR et al., 2002). Deve-se considerar, ainda, que a espécie equina foi, evolutivamente, uma espécie predada, o que se reflete em um comportamento de omissão de sinais de dor e de fuga em caso de ameaça ou perante um possível predador (SHORT, 1998). Este contexto reforça a importância do desenvolvimento de instrumentos de avaliação espécie-específicos, que possibilitem uma avaliação mais acurada da dor no equino (FLECKNELL, 2008).

A avaliação objetiva da dor consiste na mensuração de um sinal que represente de forma direta ou indireta a intensidade de dor (SILVA JÚNIOR, 2009). Taylor et al. (2002) citam como exemplos de avaliação objetiva da dor em equinos a mensuração dos níveis de catecolaminas e cortisol séricos, avaliação do grau de claudicação através da quantificação da força do membro aplicada sobre o solo, a avaliação da resposta ou reflexo à pressão 
exercida sobre uma dada região do corpo através de uma força quantificável, o método de imagem termográfica (utilizado em situações nas quais a dor esteja relacionada a uma doença ou resposta inflamatória) e a mensuração da concentração sérica de betaendorfinas, entre outros.

É importante saber que os parâmetros mensurados nas avaliações objetivas, tais como frequência cardíaca e cortisol plasmático, por exemplo, não são afetados exclusivamente pela dor, mas também por uma variedade de outros fatores, incluindo o estado de hidratação, perfusão, sepse, medo, ansiedade e uso de sedativo ou analgésico. Os dados obtidos nas avaliações objetivas devem, portanto, basear-se essencialmente em indicadores comportamentais e serem associados com o uso criterioso de sinais vitais para uma avaliação mais precisa da dor (ROBERTSON; SANCHEZ, 2010). Cabe lembrar que cada método supracitado tem seus prós e contras e situações que possibilitam ou impedem a sua aplicação, estando a sua escolha a critério do clínico veterinário.

\section{Modelos Experimentais de Dor}

Para serem eticamente aceitos, os modelos de dor experimental devem atender a critérios como: (1) o indivíduo deve estar consciente; (2) o estímulo experimental deve mimetizar um estímulo natural; (3) este estímulo deve ser minimamente invasivo, controlável, quantificável e reprodutível; e (4) a resposta para o estímulo deve ser prontamente identificada e quantificada (ROBERTSON; SANCHEZ, 2010). Estes modelos, conhecidos como testes de avaliação do limiar nociceptivo ou analgesiométricos, podem ser usados experimentalmente para avaliar a eficácia de analgésicos e clinicamente para avaliar a hiperalgesia em animais em condições de dor espontânea ou pós-operatória (LOVE et al., 2011).

Kamerling et al. (1985) descreveram um modelo de dor experimental em equinos que consiste na aplicação de um foco de luz de alta intensidade sobre a superfície cutânea, de modo a produzir um estímulo nociceptivo e determinar o limiar nociceptivo através do tempo de latência para um reflexo aversivo. Outros autores têm utilizado este método ou adaptações deste em estudos para avaliação do efeito antinociceptivo de outros agentes farmacológicos em Medicina Veterinária (ALMEIDA et al., 2005; LOVE et al., 2011; ZAMUR et 
al., 2011). A tricotomização e aplicação de tinta de cor preta sobre a região que recebe o foco de luz pode ser realizada com o objetivo de uniformizar as respostas neste modelo experimental em animais com pele que apresenta diferentes pigmentações (KAMERLING et al., 1988). Neste modelo, deve ser definido um cut-off time, isto é, um tempo máximo de exposição ao foco de luz quente, independente da apresentação ou não de uma resposta ao estímulo, de modo a evitar injúrias teciduais (LOVE et al., 2011). Alguns autores utilizando este modelo de dor experimental em equinos, adotaram um cut-off time de 10 segundos em seus ensaios (SILVA JÚNIOR, 2009; SOUZA et al., 2002; ZAMUR et al., 2011). Em vacas, Almeida et al. (2005) optaram pelo tempo limite, para emissão do foco de luz sobre a superfície cutânea, de 20 segundos.

Outros métodos são descritos para avaliação do limiar nociceptivo cutâneo em equinos. Guirro et al. (2011) utilizaram filamentos de Von Frey para determinação do limiar nociceptivo ao estímulo mecânico em equinos tratados com morfina, xilazina ou clonidina pela via epidural. Estes filamentos são instrumentos puntiformes de diâmetros variáveis que, ao exercerem pressão sobre uma determinada região, produzem um estímulo nociceptivo. Teoricamente, a força produzida pelo filamento é independente do grau de flexão do mesmo, e é determinada pela espessura da filamento. O limiar mecânico é, então, determinado pelo tamanho de filamento em que ocorra uma resposta comportamental (LOVE et al., 2011). No experimento de Guirro et al. (2011), o limiar nociceptivo foi determinado através do maior filamento incapaz de gerar uma resposta aversiva ao estímulo de uma região pós-incisada para avaliação da dor inflamatória.

O balão para cólica é um modelo de dor visceral em equinos que usa um estímulo mecânico para produzir dor experimental. Este modelo consiste na introdução de um balão no ceco através de uma fístula abdominal. O balão é posteriormente inflado, gerando um estímulo nociceptivo mecânico. Variações deste método são descritas com diferenças na localização do balão, que pode ser introduzido também em outras porções do intestino (LOVE et al., 2011; ROBERTSON; SANCHEZ, 2010). Chambers et al. (1990) desenvolveram um equipamento capaz de testar o limiar nociceptivo mecânico em equinos que funcionava utilizando ar comprimido de modo a promover um estímulo quantificável sobre a superfície 
cutânea. A pressão aplicada ao ar comprimido era calibrada através de um manômetro de mercúrio e ajustada para que o sistema exercesse uma força progressiva em uma agulha, atingindo um máximo de 27 Newtons em um minuto. 0 limiar nociceptivo era determinado através de uma resposta de retirada do membro. Outro modelo descrito para avaliação do limiar nociceptivo mecânico foi descrito por Molnar (2013), que utilizou em seus experimentos uma ferradura modificada para indução de dor solar em equinos. Este modelo consiste no uso de uma ferradura comercial soldada com uma barra em sua parte interna, a qual possui dois orifícios nos quais são inseridos parafusos e ajustados progressivamente gerando estímulo doloroso na face palmar/plantar do casco até a observação da claudicação.

Técnicas que utilizam corrente elétrica como geradora de estímulo nociceptivo também são amplamente utilizadas em equinos (LOVE et al., 2011). Com algumas variações de modelos, estas técnicas consistem, basicamente, na mensuração da menor corrente elétrica capaz de produzir uma resposta aversiva (POLYDORO, 2006).

\section{Manejo da Dor}

Por muito tempo o tratamento da dor e sua importância com relação ao bem-estar animal receberam pouco enfoque na Medicina Veterinária (BORGES, 2010). Taylor et al. (2002) citam como principais argumentos para esta relutância no tratamento da dor em equinos, as ideias de que (1) a remoção da dor de uma região do corpo já comprometida ou lesionada, como um membro, por exemplo, fará com que o animal o use ou se apoie ao mesmo em excesso, podendo causar maiores danos ao membro; (2) a existência de efeitos adversos dos fármacos utilizados; (3) a analgesia poder mascarar a evolução clínica da doença e (4) o gasto com medicação analgésica ser economicamente inviável. Estes argumentos são, em sua maior parte, inconsistentes, mas devem ser interpretados de acordo com a situação clínica a que possam se aplicar.

Atualmente, vários fármacos têm sido empregados em Medicina Veterinária para o controle e tratamento das dores aguda e crônica. Dentre eles podemos destacar os agonistas dos receptores opióides (ANDERSON; EDMONDSON, 2013; MELO JÚNIOR et al., 2012; MUIR, 2010), anestésicos locais (MUIR, 2010; ROBERTSON; SANCHEZ, 2010), anti-inflamatórios não 
esteroidais (MUIR, 2010; OLSON et al., 2015; TEIXEIRA, 2010) e agonistas dos receptores alfa-2 adrenérgicos (ANDERSON; EDMONDSON, 2013; DÓRIA et al., 2008; ENGLAND; CLARKE, 1996; GIOVANNONI et al., 2009; KAMERLING et al., 1988).

Na medicina equina, os opióides não são tão amplamente utilizados no tratamento da dor quanto em outras espécies (MUIR, 2010; ROBERTSON; SANCHEZ, 2010). Estes fármacos são, em geral, considerados analgésicos eficazes em equinos, embora o seu potencial terapêutico contraste com a capacidade de desenvolver efeitos colaterais e toxicidade em cavalos, como ataxia e hiperexcitabilidade (MUIR, 2010). Parece haver uma ligação entre tais reações adversas e a relação entre receptores opióides e seus ligantes apresentarem diferenças na distribuição e na densidade destes receptores no sistema nervoso central entre cavalos e outras espécies, mas a significância clínica desta informação ainda requer maiores esclarecimentos (MELO JÚNIOR et al., 2012; ROBERTSON; SANCHEZ, 2010). Podem predispor a constipação e altas ou repetidas dosagens podem causar retenção urinária (MUIR, 2010). Apesar disso, quando utilizados dentro dos intervalos terapêuticos recomendados, os opióides tem apresentado resultados satisfatórios (MELO JÚNIOR et al., 2012). Guirro et al. (2010) citam doses de 0,04 a 0,1 mg/kg de morfina para equinos e concluem em seus estudos que a administração intravenosa de $0,1 \mathrm{mg} / \mathrm{kg}$ do fármaco foi segura e não promoveu alterações clínicas ou comportamentais severas em cavalos da raça Puro Sangue Árabe. Robertson e Sanchez (2010) indicam um intervalo de 0,12 a 0,66 mg/kg de morfina para o tratamento de dor visceral nesta espécie e citam ainda a combinação do fármaco nestas dosagens com agentes agonistas alfa-2 adrenérgicos. O tartarato de butorfanol é um dos fármacos narcóticos mais comumente usados em animais de grande porte (ANDERSON; EDMONDSON, 2013). É classificado como um opióide agonista-antagonista, sendo capaz de produzir analgesia somática moderada e um grau levemente maior de analgesia visceral em equinos. Seu uso é indicado nas doses de 0,02 a 0,1 mg/kg por via intravenosa ou intramuscular ou em infusão contínua intravenosa a uma taxa de 13 a $23 \mathrm{mcg} / \mathrm{kg} / \mathrm{hora}, 15$ minutos após um bolus inicial de $18 \mathrm{mcg} / \mathrm{kg}$ (ROBERTSON; SANCHEZ, 2010). Outro ponto positivo no uso de agonistas opióides inclui sua administração por via epidural, a qual promove analgesia isenta de depressão respiratória, hipomotilidade, hipertermia ou alterações locomotoras. Por esta via, Guirro et al. (2011) observaram analgesia de 24 horas 
após anestesia epidural com 0,1 mg/kg de morfina. Uma grande vantagem no uso destes fármacos é o fato de possuírem antagonistas conhecidos, como a naloxona, por exemplo, que possibilita a reversão dos efeitos clínicos dos agonistas opióides (MUIR, 2010).

O tramadol é considerado um fraco agonista do receptor opióide $\mu$, possui também efeitos relacionados à inibição da recaptação de noradrenalina e serotonina, aumentando seu poder analgésico (MOLNAR, 2013). Silva Júnior (2009), avaliando os efeitos do tramadol em equinos, na dose de $3 \mathrm{mg} / \mathrm{kg}$, xilazina na dose de $0,5 \mathrm{mg} / \mathrm{kg}$, ou da associação de ambos, concluiu que o uso isolado do tramadol na dose supracitada não promove analgesia somática, embora também não provoque alterações comportamentais significativas. $O$ autor conclui ainda que a associação de tramadol e xilazina nas doses citadas acima também não compõem uma boa opção visando a sedação e a analgesia em equinos. Em contrapartida, outros autores relatam a ocorrência de ataxia, sudorese e/ou tremores musculares com a administração de $2 \mathrm{mg} / \mathrm{kg}$ de tramadol por via intravenosa (SHILO et al., 2008). Melo Júnior et al. (2012) associam a menor efetividade analgésica do tramadol em equinos quando comparada à analgesia obtida no cão e no gato a particularidades da biotransformação do fármaco pelos equinos, uma vez que seus metabólitos desempenham papel importante na antinocicepção.

Os anestésicos locais estão entre os fármacos analgésicos mais empregados na cirurgia de animais de produção (ANDERSON; EDMONDSON, 2013). Estes medicamentos produzem analgesia pelo bloqueio de canais de sódio, evitando, portanto, a iniciação e a condução dos impulsos elétricos pelos nociceptores aferentes (MUIR, 2010). Além dos fins cirúrgicos, a anestesia local é também comumente utilizada durante o exame clínico do aparelho locomotor para investigar a origem da claudicação e os sítios de dor em equinos (SILVA et al., 2015). Várias técnicas possibilitam o uso destes fármacos, tais como bloqueios locais infiltrativos, bloqueios perineurais, bloqueios regionais (paravertebral e epidural, por exemplo), e bloqueios regionais intravenosos entre garrotes (torniquetes), entre outras (ANDERSON; EDMONDSON, 2013). A bupivacaína é o anestésico local de longa duração mais comumente utilizado na rotina clínica e anestesiológica da medicina equina, podendo atingir um efeito de duração de 180 a 480 minutos, com latência de 10 a 30 minutos (ESCODRO et 
al., 2015). O uso da lidocaína em infusão contínua por via intravenosa é também descrito como adjuvante na analgesia em anestesias gerais. A infusão de lidocaína a uma taxa de 50 $\mathrm{mcg} / \mathrm{kg} / \mathrm{minuto}$, após um bolus de $2,5 \mathrm{mg} / \mathrm{kg}$, é capaz de reduzir a concentração alveolar mínima (CAM) do isoflurano em até $25 \%$ nos equinos sem que o ocorra o aparecimento de efeitos adversos (DZIKITI et al., 2003).

Os anti-inflamatórios não esteroidais (AINEs) possuem suas propriedades analgésicas e antiinflamatórias bastante documentadas. Em contrapartida, os efeitos adversos sobre o trato gastrointestinal e renal, como ulcerações gástricas, cólicas e necrose tubular, têm sido descritos (ROBERTSON; SANCHEZ, 2010). A analgesia e o efeito anti-inflamatório são produzidos pela redução da síntese de prostaglandinas através da inibição das ciclooxigenases (ANDERSON; EDMONDSON, 2013). As prostaglandinas e leucotrienos são peças-chave na produção da sensibilização periférica e provavelmente no aumento da sensibilização central (MUIR, 2010). Frequentemente, os AINEs são associados com agentes opióides para controle da dor pós-operatória (SILVA et al., 2015). Os efeitos dos AINEs na síntese de prostaglandinas tanto periféricas quanto centrais sugerem que estes fármacos possuem um importante papel em protocolos de analgesia multimodal (COETZEE, 2013). Robertson e Sanchez (2010) destacam o flunixin meglumine como um dos fármacos mais importantes e mais comumente usados para o tratamento da dor visceral em equinos e recomendam o uso de doses de 0,25 a 1,1 mg/kg por via intravenosa ou oral, porém ressaltam que deve-se evitar as doses mais elevadas em intervalos menores que 12 horas. 0 cetoprofeno é indicado pelos mesmos autores em administração intravenosa na dose de 2,2 $\mathrm{mg} / \mathrm{kg}$ a cada 24 horas e a fenilbutazona em doses de 2,2 a $4,4 \mathrm{mg} / \mathrm{kg}$ em administração oral ou intravenosa a cada 12 ou 24 horas.

Sabe-se que a norepinefrina participa da modulação das respostas relacionadas à dor através de várias vias. Os agonistas alfa-2 adrenérgicos tem um perfil analgésico bem estabelecido e têm sido amplamente empregados como adjuvantes em associação com anestésicos e analgésicos em quadros perioperatórios (GIOVANNONI et al., 2009). Este grupo de fármacos engloba princípios ativos como a xilazina, frequentemente usada em doses de 0,2 a $1,1 \mathrm{mg} / \mathrm{kg}$ por via intravenosa ou intramuscular; a detomidina, usada em 
intervalos terapêuticos de 0,005 a 0,04 $\mathrm{mg} / \mathrm{kg}$ por via intravenosa ou intramuscular; e a medetomidina, indicada para uso intravenoso nas doses de 0,004 a 0,01 mg/kg (ROBERTSON; SANCHEZ, 2010). Os efeitos clínicos decorrentes da administração de agonistas alfa-2 adrenérgicos, em geral, incluem sedação, bradicardia, depressão respiratória e, raramente, excitação (MUIR, 2010). Podemos destacar dentre as maiores desvantagens de todos os agonistas alfa-2 adrenérgicos o decréscimo da motilidade gastrointestinal, que é acompanhado de bradicardia e uma hipertensão transitória, seguida de hipotensão (ENGLAND; CLARKE, 1996; ROBERTSON; SANCHEZ, 2010). A xilazina é bastante conhecida por promover sedação e analgesia tanto para exames diagnósticos quanto para o tratamento da dor visceral em cavalos (ROBERTSON; SANCHEZ, 2010). Estes fármacos também podem ser administrados por via epidural, a xilazina por esta via produz analgesia perineal maior que quando administrada por via intramuscular (GIOVANNONI et al., 2009). Cabe ressaltar que estudos investigativos realizados com a detomidina demonstraram que a concentração plasmática necessária para obtenção de analgesia foi cerca de 10 vezes maior que a concentração requerida para a sedação, o que alerta para o fato de que, em se tratando de agonistas alfa-2 adrenérgicos, a ocorrência de sedação não corresponde à analgesia (ROBERTSON; SANCHEZ, 2010). loimbina, idazoxan, tolazolina e o atipamezole são antagonistas alfa-adrenérgicos e seu uso clínico permite a reversão dos efeitos farmacológicos descritos (ENGLAND; CLARKE, 1996; MUIR, 2010).

A cetamina, em doses subanestésicas, produz antinocicepção somática e possui um papel importante na prevenção da sensibilização central. Este efeito é obtido através do antagonismo aos receptores N-metil-D-aspartato. A infusão contínua intravenosa de 0,4 a $1,2 \mathrm{mg} / \mathrm{kg} / \mathrm{hora}$ é utilizada em protocolos de analgesia visceral, porém é possível que ocorra hiperexcitabilidade com o uso de doses elevadas (ROBERTSON; SANCHEZ, 2010). A gabapentina também tem sido usada em cavalos para promover analgesia complementar, mas sua eficácia tem sido discutida (CALDWELL et al., 2015; MUIR, 2010). Em vacas, a cetamina a uma concentração de 5\%, quando aplicada por via epidural em um volume de 20 $\mathrm{mL}$, promoveu ataxia por cerca de 40 minutos e analgesia ao longo de 60 minutos, sem alterar as frequências cardíaca e respiratória, a temperatura retal, motilidade ruminal e a pressão arterial média ao longo do estudo (ANDERSON; EDMONDSON, 2013). 
Finalmente, outras opções terapêuticas alternativas e complementares ao manejo da dor em equinos incluem a acupuntura, quiropraxia e a homeopatia, entre outras, destacando-se como adjuvantes nos protocolos farmacológicos convencionais (MUIR, 2010).

\section{CONCLUSÃO}

A dor é uma condição que tem impacto direto na qualidade de vida de um animal e seu reconhecimento por parte do Médico Veterinário é um ponto-chave para a excelência no seu controle e tratamento. O entendimento sobre seus aspectos fisiopatológicos é fundamental para a escolha do tratamento mais adequado, uma vez que os mecanismos de ação dos diferentes medicamentos disponíveis podem ser mais ou menos eficientes, de acordo com a origem e a intensidade da dor. O desenvolvimento de novos modelos experimentais de dor e o aperfeiçoamento dos métodos já existentes é também um importante desafio, pois permitirão avaliar com precisão cada vez maior a qualidade analgésica dos protocolos farmacológicos utilizados, contribuindo diretamente para um melhor aproveitamento na terapêutica do controle da dor em equinos.

\section{CLINICAL AND EXPERIMENTAL FEATURES OF PAIN IN HORSES: REVIEW}

\section{ABSTRACT}

$\mathrm{P}$ ain is a phenomenon produced from a sensorial or emotional aversive experience, represented by a conscious real or potential tissue damage that generates several physiological and behavioral changes, which avoid or minimize the occurrence of such damage, aiming to protect the organism and maintain the homeostasis. This phenomenon has a direct impact over the animal welfare and its control is a professional responsibility of the Veterinarian. Pain perception by the animal depends of several events that involve the peripheral and central nervous system, such as transduction, transmission, modulation, projection and central processing of the electric impulse generated by the harmful stimulus. Currently, several drugs are available to control pain in equine medicine and various experimental methods are described to study pain in this species. These models allow the evaluation of the efficacy of analgesic drugs and deepen the comprehension about the mechanisms involved in pain physiopathology. This article aims to approach the main challenges in the study of pain in horses, under experimental and clinical points of view. 
Keywords: Pain. Analgesia. Nociception. Experimental Models.

\section{ASPECTOS CLÍNICOS Y EXPERIMENTALES DEL DOLOR EN LOS EQUINOS: REVISIÓN DE LA LITERATURA}

\section{RESUMEN}

$\mathrm{E}$ I dolor es un fenómeno producido a partir de una experiencia sensorial o emocional aversiva, representada por la conciencia de un daño tisular real o potencial, lo que arroja una serie de cambios fisiológicos y de comportamiento que tienen como objetivo prevenir o minimizar la ocurrencia de tales daños en el intuido de proteger el cuerpo y mantener la homeostasis. Este fenómeno tiene un impacto directo en el bienestar de los animales y su control es una responsabilidad profesional del Veterinario. La percepción del dolor por el individuo depende de una serie de eventos que involucran el sistema nervioso central y periférico, tales como la transducción, la transmisión, la modulación, la proyección y el procesamiento central del pulso eléctrico generado por los estímulos nocivos. En la actualidad, varias drogas están disponibles para su empleo en el control del dolor en la medicina equina y diversos métodos experimentales se describen para el estudio del dolor en esta especie. Estos modelos permiten la evaluación de la eficacia de fármacos analgésicos y profundizar la comprensión de los mecanismos implicados en la fisiopatología del dolor. El propósito de este artículo es abordar los principales desafíos en el estudio del dolor en los caballos, en virtud de los puntos de vista experimentales y clínicos.

Palabras clave: Dolor. Analgesia. Nocicepción. Modelos Experimentales.

\section{REFERÊNCIAS}

ALMEIDA, R. M.; VALADÃO, C. A. A.; FARIAS, A.; DUQUE, J. C. M.; SOUZA, A. H. Avaliação dos efeitos do amitraz por via epidural em vacas. Brazilian Journal of Veterinary Research and Animal Science, v. 42, n. 6, p. 419-428, 2005.

ANDERSON, D. E.; EDMONDSON, M. A. Prevention and Management of Surgical Pain in Cattle. Veterinary Clinics of North America: Food Animal Practice, v. 29, n. 1, p. 157-184, 2013.

BORGES, T. D. Ensino da relevância da dor para o bem-estar animal: Panorama em cursos de Medicina Veterinária no Brasil e proposta de curso à distância. Curitiba, UFPR, 2010. 119p. Dissertação (Mestrado em Ciências Veterinárias), Programa de Pós-Graduação em Ciências Veterinárias, Universidade Federal do Paraná, 2010. 
CALDWELL, F. J.; TAINTOR, J.; WAGUESPACK, R. W.; SELLERS, G.; JOHNSON, J.; LIN, H. Effect of $\mathrm{PO}$ administered gabapentin on chronic lameness in horses. Journal of Equine Veterinary Science, v. 35, n. 6; p. 536-540, 2015.

CASTRO, A. B. Anatomia das vias nociceptivas. In: SIMBIDOR - SIMPÓSIO BRASILEIRO E ENCONTRO INTERNACIONAL SOBRE DOR, 8, 2007, São Paulo. ARQUIVOS. São Paulo: Office Editora, 2007. p. 23-28.

CHAMBERS, J. P.; LIVINGSTON, A.; WATERMAN, A. E. A device for testing nociceptive thresholds in horses. Veterinary Anaesthesia and Analgesia, v. 17, n. 1, p. 42-44, 1990.

COETZEE, J. F. A review of analgesic compounds used in food animals in the United States. Veterinary Clinics of North America: Food Animal Practice, v. 29, p. 11-28, 2013.

DIZIKITI, T. B.; HELLEBREKERS, L. J.; VAN DIJK, P. Effects of intravenous lidocaine on isoflurane concentration, physiological parameters, metabolic parameters and stress-related hormones in horses undergoing surgery. Journal of Veterinary Medicine. A, Physiology, Pathology, Clinical Medicine, v. 50, n. 4, p. 190-195, 2003.

DÓRIA, R. G. S.; VALADÃO, C. A. A.; DUQUE, J. C.; FARIAS, A.; ALMEIDA, R. M.; NETTO, A. C. Comparative study of epidural xylazine or clonidine in horses. Veterinary Anaesthesia and Analgesia, v. 35, p. 166-172, 2008.

ENGLAND, G. C. W.; CLARKE, K. W. Alpha2 adrenoceptor agonists in the horse - A review. British Veterinary Journal, v. 152, n. 6, p. 641-657, 1996.

ESCODRO, P. B.; OLIVEIRA, C. F.; TONHOLO, J.; NASCIMENTO, T. G.; VILANI, R. G. O.; BRUHN, F. R. P. Estudo comparativo entre bupivacaína $0,5 \%$ e ropivacaína $0,5 \%$ no bloqueio dos nervos palmares de equinos hígidos. Archives of Veterinary Science, v. 20, n. 2, p. 67-75, 2015.

FLECKNELL, P. Analgesia from a veterinary perspective. British Journal of Anaesthesia, v. 101, n. 1, p. 121-124, 2008.

GIOVANNONI, M. P.; GHELARDINI, C.; VERGELLI, C.; DAL PIAZ, V. Alpha2-Agonists as Analgesic Agents. Medicinal Research Reviews, v. 29, n. 2, p. 339-368, 2009.

GRAUW, J. C.; VAN LOON, J. P. A. M. Systematic pain assessment in horses. The Veterinary Journal, v. 209, p. 14-22, 2016, http://dx.doi.org/10.1016/j.tvjl.2015.07.030

GUIRRO, E. C. B. P.; PEROTTA, J. H.; VALADÃO, C. A. A. Efeitos clínicos e comportamentais promovidos pela injeção intravenosa de morfina em equinos Puro Sangue Árabe. Ciência Animal Brasileira, v. 11, n. 4, p. 853-859, 2010. 
GUIRRO, E. C. B. P.; SOBRINHO, G. R.; FERREIRA, I. M. M.; VALADÃO, C. A. A. Efeitos comportamental, clínico e analgésico promovidos pela injeção epidural preventiva de morfina, xilazina ou clonidina, em equinos. Ciência Rural, Santa Maria, v. 41, n. 10, p. 17901796, 2011.

KAMERLING, S. G.; CRAVENS, W. M. T.; BAGWELL, C. A. Objective assessment of detomidineinduced analgesia and sedation in the horse. European Journal of Pharmacology, v. 151, p. 1-8, 1988.

KAMERLING, S. G.; WECKMAN, T. J.; DEQUICK, D. J.; TOBIN, T. A method of studying cutaneous pain perception and analgesia in horses. Journal of Pharmacological Methods, $\mathrm{v}$. 13, p. 267-274, 1985.

KLAUMANN, P. R.; WOUK, A. F. P. F.; SILLAS, T. Patofisiologia da dor. Archives of Veterinary Science, v. 13, n. 1, p. 01-12, 2008.

LE BARS, D.; GOZARIU, M.; CADDEN, S. W. Animal Models of Nociception. Pharmacological Reviews, v. 53, n. 4, p. 597-652, 2001.

LOVE, E. J.; MURRELL, J.; WHAY, H. R. Thermal and mechanical nociceptive threshold testing in horses: A review. Veterinary Anaesthesia and Analgesia, v. 38, p. 03-14, 2011.

MELO JÚNIOR, J.; MATTOS JÚNIOR, E.; WILGES, C. H. M.; FRANCO, L. G. Controle da dor em animais de grande porte: Perspectivas para o uso do tramadol. Enciclopédia Biosfera, Goiânia, v. 8, n. 15, p. 502-516, 2012.

MOLNAR, B. F. P. Avaliação dos efeitos analgésicos do tramadol administrado pela via oral e intramuscular na espécie equina (Equus caballus) utilizando modelo de ferradura modificada para indução de dor solar. Porto Alegre, UFRGS, 2013. 40p. Dissertação (Mestrado em Medicina Animal), Universidade Federal do Rio Grande do Sul, 2013.

MUIR, W. W. Pain: Mechanisms and Management in Horses. Veterinary Clinics of North America: Equine Practice, v. 26, p. 467-480, 2010.

OLSON, M. E.; FIERHELLER, E.; BURWASH, L.; RALSTON, B.; SCHATZ, C.; MATHESON-BIRD, H. The efficacy of meloxicam oral suspension for controlling pain and inflammation after castration in horses. Journal of Equine Veterinary Science, v. 35, p. 724-730, 2015.

POLYDORO, A. S. Estudo "in vitro" e "in vivo" da administração subaracnóide de opióides hiperbáricos em cavalos. Santa Maria, UFSM, 2006. 79p. Tese (Doutorado em Cirurgia Veterinária), Universidade Federal de Santa Maria, 2006.

ROBERTSON, S. A.; SANCHEZ, L. C. Treatment of Visceral Pain in Horses, Veterinary Clinics of North America: Equine Practice, v. 26, p. 603-617, 2010. 
SHILO, Y.; BRITZI, M.; EYTAN, B.; LIFSHITZ, T.; SOBARACK, S.; STUMMAN, A. Pharmacokinetics of tramadol in horses after intravenous, intramuscular and oral administration. Journal of Veterinary Pharmacology and Therapeutics, v. 31, n. 1, p. 60-65, 2008.

SHORT, C. E. Fundamentals of pain perception in animals. Applied Animal Behaviour Science, v. 59, n. 1, p. 125-133, 1998.

SILVA, G. B.; DE LA CÔRTE, F.; BRASS, K. E.; AZEVEDO, M. S.; DAU, S.; CENI, F.; LOPES, L. F. D. Duration and efficacy of different local anesthetics on the palmar digital nerve block in horses. Journal of Equine Veterinary Science, v. 35, p. 749-755, 2015.

SILVA JÚNIOR, J. R. Efeitos do tramadol isolado ou associado à xilazina em equinos. Jaboticabal, UNESP, 2009. 85p. Tese (Doutorado em Clínica Médica Veterinária), Universidade Estadual Paulista Júlio de Mesquita Filho, 2009.

SOUZA, A. H.; VALADÃO, A. A.; QUEIROZ-NETO, A.; ZAMUR, G.; COELHO, V. Efeito da injeção subcutânea de doses baixas de cetamina em equinos. Ars Veterinária, Jaboticabal, v. 18, n. 3, p. 223-230, 2002.

TAYLOR, P. M.; PASCOE, P. J.; MAMA, K. R. Diagnosing and treating pain in the horse. Where are we today? The Veterinary Clinics: Equine Practice, v. 18, p. 1-9, 2002.

TEIXEIRA, M. J. Fisiopatologia da Nocicepção e da Supressão da Dor. Jornal Brasileiro de Oclusão, ATM e Dor Orofacial, v. 1, n. 4, p. 329-334, 2001.

TEIXEIRA, F. M. Avaliação comparativa do efeito de fármacos anti-inflamatórios com a acupuntura no modelo de dor pós-incisional em ratos. Seropédica, UFRRJ, 2010. 77p. Dissertação (Mestrado em Ciências Veterinárias), Universidade Federal Rural do Rio de Janeiro, 2010.

ZAMUR, G.; ARAÚJO, A.; MATAQUEIRO, M. I.; FERRAZ, G. C.; QUEIROZ-NETO, A. Comparação dos efeitos sedativos e/ou antinociceptivos dos tranquilizantes acepromazina, levomepromazina e azaperone em equinos. Ars Veterinária, Jaboticabal, v. 27, n. 4, p. 231240, 2011.

Autor para correspondência: José Edgard de Oliveira Alves. Universidade Estadual do Norte Fluminense, Hospital Veterinário, Campos dos Goytacazes (RJ), CEP 28013-602, Brasil. edgardoliveiraalves@hotmail.com 